\title{
Application of Neural Networks to the Optimization of the Thermal Treatment Process of Wood Materials
}

\author{
M. Hadjiski, N. Deliiski, A. Grancharova
}

Key Words: Wood thermal treatment process; optimization; neural networks.

\begin{abstract}
In this paper, a software system for 2-dimensional and 3-dimensional modeling of frozen and not frozen logs and prismatic wood materials is used as a basis for optimization of the thermal treatment process of such materials. Then, the dependence of the optimal heating time on various parameters is approximated by means of neural networks. This would allow a fast real-time computation of the optimal heating time instead of performing extensive numerical simulations of the model comprised of partial differential equations.
\end{abstract}

\section{Introduction}

A central objective in management, control and operation of the one of the most heat- and time-consuming processes in transforming the raw material into an industrial product - the wood thermal treatment process (TTP) - is increasing the competition, reducing the heat energy consumption, growing the capacity, and fulfilling the strict tight technological requirements.

Thermal treatment of wood materials comprises spatially distributed technological processes. They are described by partial differential equations (PDE). Various approaches have been developed during the last half century in order to overcome the numerous difficulties in modeling, simulation and control arising from the comparison with the lumped parameter systems (e.g. [1]). The academic-oriented research created principally new directions in transforming the infinite space presentation by finite dimension modeling in the linear case (e.g. [2]). The investigations have been enhanced towards nonlinear distributed parameter systems modeling and control $[3,4]$.

During the last decade several intelligent techniques have been incorporated in modeling and model-predictive control of distributed parameter systems [5], and especially the implementation of artificial neural networks (NN) [6]. Though that wood thermal treatment processes were deeply studied in different technological aspects (e.g. [7]), only a few investigations are addressed to modeling and control of TTP using the listed above achievements in the area of distributed parameter systems. Available results are formed mainly in modeling [8,9].

In this paper, the earlier developed system for $2 \mathrm{D}$ and
$3 \mathrm{D}$ modeling of frozen and not frozen logs and prismatic wood materials $[10,11,12]$ is used as a basis for optimization of the thermal treatment process by means of neural networks.

\section{Mathematical Modeling of the Thermal Treatment Process}

\section{TTP Principles and Peculiarities}

The thermal treatment is a periodical process of wood material heating in order to reach a given average mass temperature of the charge subjected to prescribed requirements according to the admissible surface temperature and the internal temperature gradients. TTP is carried out in autoclaves with steam as a heating agent or in pits using hot water. The cross-section of a typical autoclave is presented in figure 1.

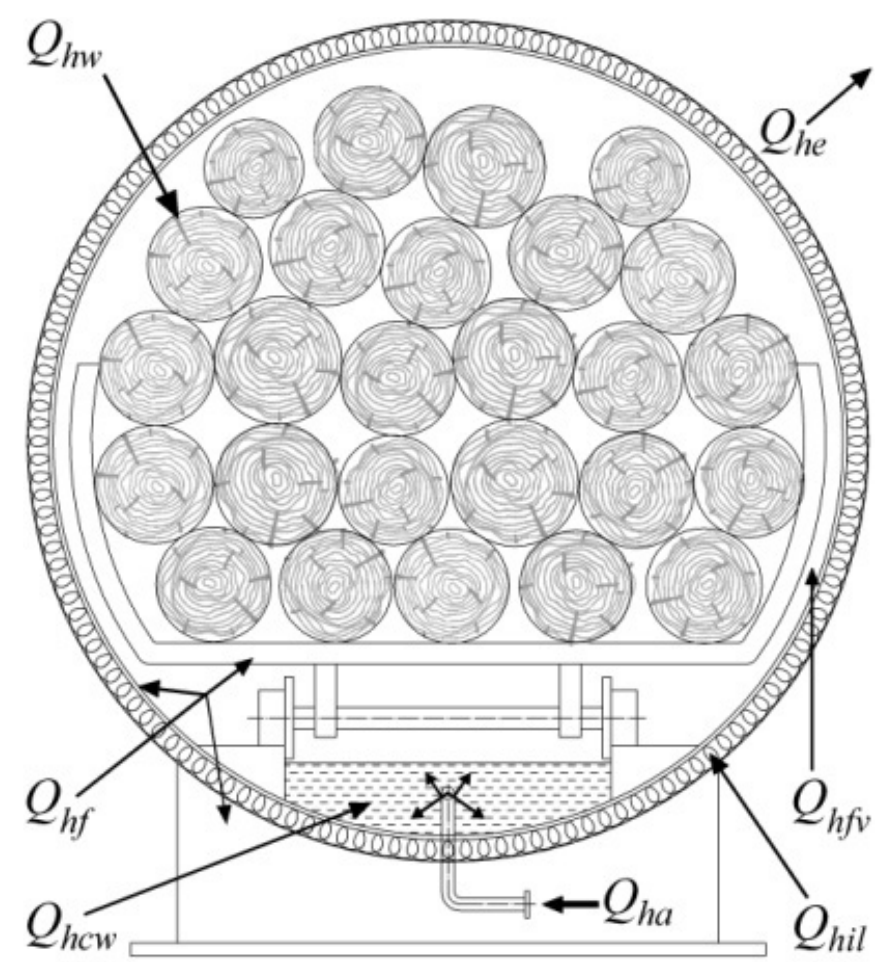

Figure 1. Autoclave cross-section 


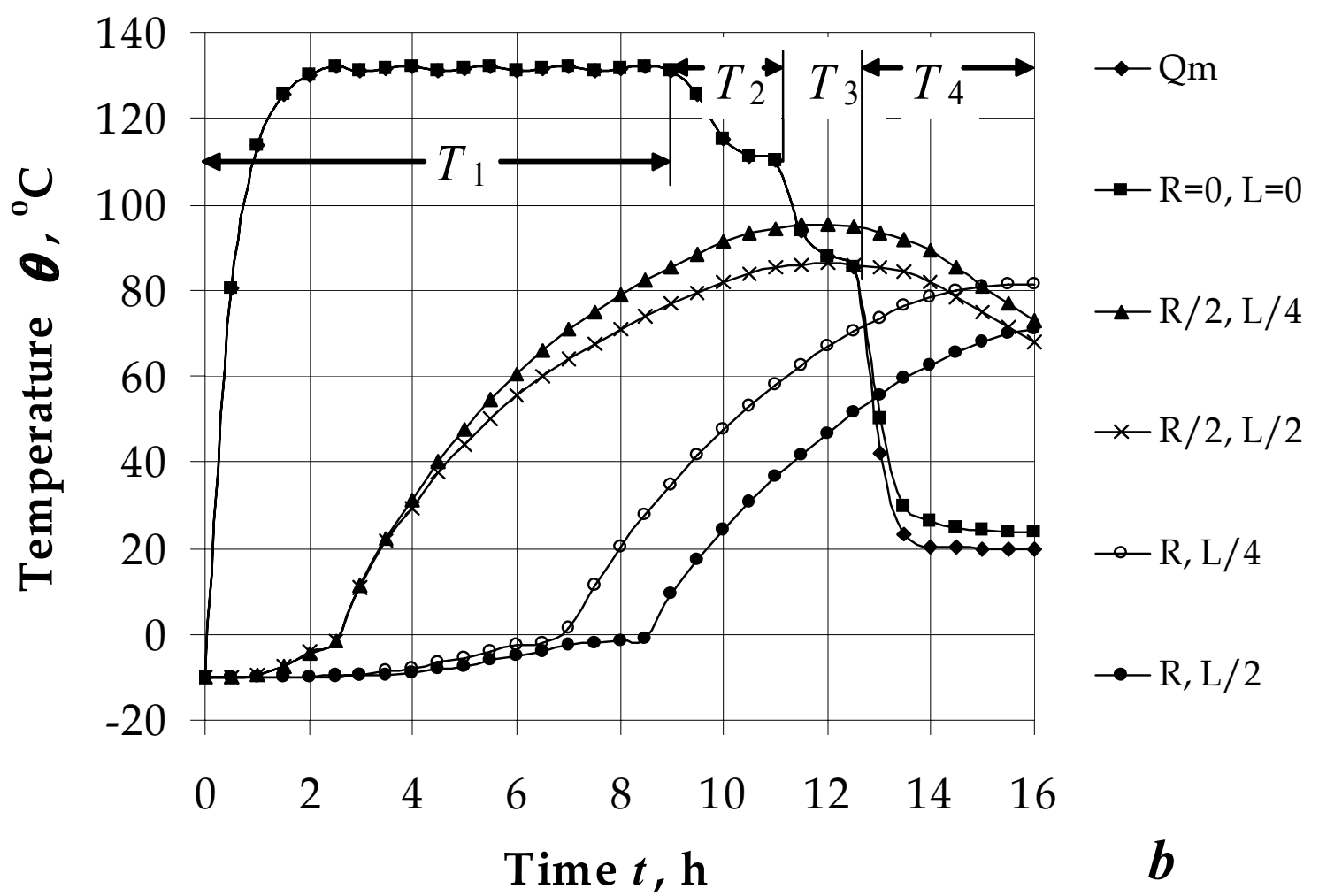

Figure 2. Temperature time profiles during TTP for frozen beech logs

The thermal treatment is a spatiotemporal nonlinear process. Typical temperature profiles for some characteristic points of the cross-section of beech logs with an initial temperature $-10^{\circ} \mathrm{C}$ during their steaming in an autoclave with diameter of $2.4 \mathrm{~mm}$ and length of $9.0 \mathrm{~m}$, obtained by computer simulation [12] are presented in figure 2. Two distinct heating processes can be observed in figure 2: $(i)$ fast for the external heating medium temperature $\theta_{m}$ and (ii) slow for the internal points of the wood space.

The only temperature of the heating agent $\theta_{m}$ and the steam flow rate $F_{m}$ can be measured on-line. Due this strong drawback the only model-based approaches are suitable for control systems design, state estimation, dispatching operations, and production scheduling. Unfortunately the lack of measurements provokes strong difficulties in the TTP management.

The operational conditions of each charge can be different because of the considerable variations of the initial conditions - wood specie, size, moisture content, temperature of the wood and aggregate state of the water in it at the beginning of TTP, and the relative loading of the autoclave as well. At the same time some of these initial charging parameters are immeasurable. Unfortunately they determine the parameters, the initial and boundary conditions necessary to solve the first-principle models based on partial differential equations (PDE) $[10,11,12]$. The attempt to use as model parameters some 'average' or operator's given values causes models fail and discredit all efforts for advanced control implementation in real industrial conditions.
The TTP has the following peculiarities:

- Only $\theta_{m}$ and $F_{m}$ are on-line available as a secondary (indirect measurements).

- The values of $\theta_{m}$ and $F_{m}$ are available only less than $20 \%$ of the whole TTP duration.

- The initial changing conditions are immeasurable.

- The accuracy of the PDE-based model considerably depends on the initial changing conditions.

- The TTP is strongly nonlinear distributed parameter system, especially in dependence of the frozen/unfrozen charged timber.

- The mathematical TTP modeling is highly timeconsuming due to the nonlinearities and the space distribution. Thus adopting the on-line calculations as a part of the control algorithm cannot be a relevant strategy. More appropriate would be:

- To develop models with reduced dimensionality;

- The biggest part of the calculations to be carried out off-line;

- To accept a strategy of suboptimal control.

The TTP is imposed on numerous constraints like [5]

$$
\frac{\partial \theta(x, y, z, t)}{\partial t}<\Gamma_{1}, \frac{\partial \theta(x, y, z, t)}{\partial n}<\Gamma_{2}, \theta(s)<\theta^{\max },
$$

where $\theta(x, y, z, t)$ is the wood temperature in the point with coordinates $(x, y, z), t$ - time, $n$ - the normal vector, $\theta(s)$ - the surface temperature, $s$ - the surface coordinates $\Gamma_{1}, \Gamma_{2}, \theta^{\max }-$ the technological requirements.

- The TTP control system constraints depend strongly on the model parameters. 
In addition to the uncertainty due to the immeasurable initial charging parameters, the TTP is dependent on some specific bio- and morphological properties of the timber. They are immeasurable and stochastic [7].

\section{Mathematical Modeling of TTP}

A basic task in heat modeling, developing and managing the technologies and control of TTP is the determination of the temperature in certain points from the volume of the wood materials, at any moment from the process of their heating and further conditioning in aerial medium.

During the heating of the wood materials along with the purely thermal processes, a mass-exchange occurs between the heating medium and the wood. The values of the moisture diffusion of the different wood species cross sectional to their fibers are hundreds of times smaller than the values of their temperature conductivity. In a longitudinal to the fibers direction, the temperature conductivity exceeds the moisture diffusion by more than a hundred times.

These facts determine not so big change in the content of water in the materials during their TTP, which lags significantly from the distribution of heat in them. This allows during the creation of a mathematical model to disregard the exchange of mass between the wood and the heating medium and the change in temperature in the materials to be viewed as a result of a purely thermo-exchange process, where the heat in them is distributed only through thermo-conductivity.

The process of heating the subjected to TTP wood materials with prismatic form can be modeled with the help of the following equation:

$$
\begin{gathered}
c\left(\Theta, w, w_{f s p}\right) \cdot \rho\left(\rho_{b}, w\right) \frac{\partial \Theta(x, y, z, t)}{\partial t}= \\
\frac{\partial}{\partial x}\left[\lambda_{x}\left(\Theta, w, w_{f s p}, \rho_{b}\right) \frac{\partial \Theta(x, y, z, t)}{\partial x}\right]+ \\
\frac{\partial}{\partial y}\left[\lambda_{y}\left(\Theta, w, w_{f s p}, \rho_{b}\right) \frac{\partial \Theta(x, y, z, t)}{\partial y}\right]+ \\
\frac{\partial}{\partial z}\left[\lambda_{z}\left(\Theta, w, w_{f s p}, \rho_{b}\right) \frac{\partial \Theta(x, y, z, t)}{\partial z}\right]
\end{gathered}
$$

with an initial condition

(3) $\Theta(x, y, z, 0)=\Theta_{0}$ and the following boundary conditions:

- during TTP:

(4) $\Theta(0, y, z, t)=\Theta(x, 0, z, t)=\Theta(x, y, 0, t)=\Theta_{m}(t)$

- during the conditioning in aerial medium of the heated materials:

(5)

$$
\begin{aligned}
& \text { (5) } \frac{\partial \Theta(0, y, z, t)}{\partial x}=-\frac{\alpha_{s x}(0, y, z, t)}{\lambda_{s x}(0, y, z, t)}\left[\Theta(0, y, z, t)-\Theta_{a}\right] \\
& \text { (6) } \frac{\partial \Theta(x, 0, z, t)}{\partial y}=-\frac{\alpha_{s y}(x, 0, z, t)}{\lambda_{s y}(x, 0, z, t)}\left[\Theta(x, 0, z, t)-\Theta_{a}\right]
\end{aligned}
$$

$$
\frac{\partial \Theta(x, y, 0, t)}{\partial z}=-\frac{\alpha_{s z}(x, y, 0, t)}{\lambda_{s z}(x, y, 0, t)}\left[\Theta(x, y, 0, t)-\Theta_{a}\right],
$$

where $\Theta, \Theta_{0}, \Theta_{m}, \Theta_{a}$, are the temperature, temperature of the wood at the beginning of TTP, temperature of the processing medium during TTP, and temperature of the aerial medium near the subjected to conditioning heated wood materials respectively, $\mathrm{K}$. The meaning of the other notation is as follows:

$c$ - specific heat capacity of the wood, $\mathrm{J}_{\mathrm{kg}} \mathrm{kg}^{-1} \cdot \mathrm{K}^{-1}$;

$w$ - moisture content of the wood, kg. $\mathrm{kg}^{-1}$;

$w_{f s p}$ - fiber saturation point of the wood specie, $\mathrm{kg}^{\mathrm{kg}} \mathrm{kg}^{-1}$;

$\lambda_{x}^{f s p}, \lambda_{y}$ and $\lambda_{z}-$ thermal conductivities of the wood in radial, tangential and longitudinal anatomical directions respectively, $\mathrm{W} \cdot \mathrm{m}^{-1} \cdot \mathrm{K}^{-1}$;

$\lambda_{s x}, \lambda_{s y}$ and $\lambda_{s z}-$ thermal conductivities on the surfaces of the wood materials in radial, tangential and longitudinal directions respectively, $\mathrm{W} \cdot \mathrm{m}^{-1} \cdot \mathrm{K}^{-1}$;

$\rho-$ density of the wood, kg.m ${ }^{-3}$;

$\rho_{b}$ - basic density of the wood, equal to the dry mass divided by green volume, $\mathrm{kg} \cdot \mathrm{m}^{-3}$;

$\alpha_{s x}, \alpha_{s y}$ and $\alpha_{s z}-$ heat transfer coefficients between the respective surfaces (perpendicular to the coordinate axes $x, y, z$ ) of the subjected to TTP wood materials and the surrounding aerial medium, W. $\mathrm{m}^{-2} \cdot \mathrm{K}^{-1}$;

$x$ - linear coordinate of each point from the thickness of the wood prism, m;

$y$ - linear coordinate of each point from the width of the wood prism, m;

$z$ - linear coordinate of each point from the length of the wood prism, m;

$t$ - time, s.

For the solution of the created mathematical model a software package has been developed in the computing medium of Visual Fortran Professional. Through simulation of computer experiments this package allows to investigate the distribution of the temperature field in the volume of wood materials at different initial and boundary conditions and to form scientifically based technologies and control for TTP both in autoclaves and equipments, working under atmospheric pressure.

The vector $p$ of immeasurable initial charging parameters has the following four components:

(8) $p=p\left(d, w_{0}, \theta_{0}, \gamma\right)$

where $d$ is the equivalent size, $w_{0}, \theta_{0}$ are the initial moisture content and the temperature, $g$ is the relative timber loading charged in the autoclave. All these components are arguments for the dynamic parameters $\lambda_{x}, \lambda_{y}, \lambda_{t}, \rho, \rho_{b}, c$ in equation (2), the initial condition (3) and the boundary conditions (4)-(7). The heat accumulation in the autoclave can be presented in a semi-explicit form:

$$
\text { (9) } \frac{d \theta_{m}(t)}{d t}=F_{m}\left(\theta_{m}(t), u(t), p, b\right), t<T_{m},
$$

where $F_{m}$ is a nonlinear function, $b$ is a known vector of physical constants and constructive parameters. 
The variations of charging conditions represented by the parametric vector $p$ influence the both interrelated TTP parts as shown in figure 3 , where $\pi$ is the wood specie.

The dynamic behavior of the medium temperature $\theta_{m}(t)$ must be defined by simultaneous solving this intercon- nected system of differential and algebraic equations. The solution in the time interval $0 \leq t \leq T_{m}$ strongly depends on the parameter-vector $p$ values and it is the only available sensor information in real operations usable for the $p$ reconstruction which we use in this research.

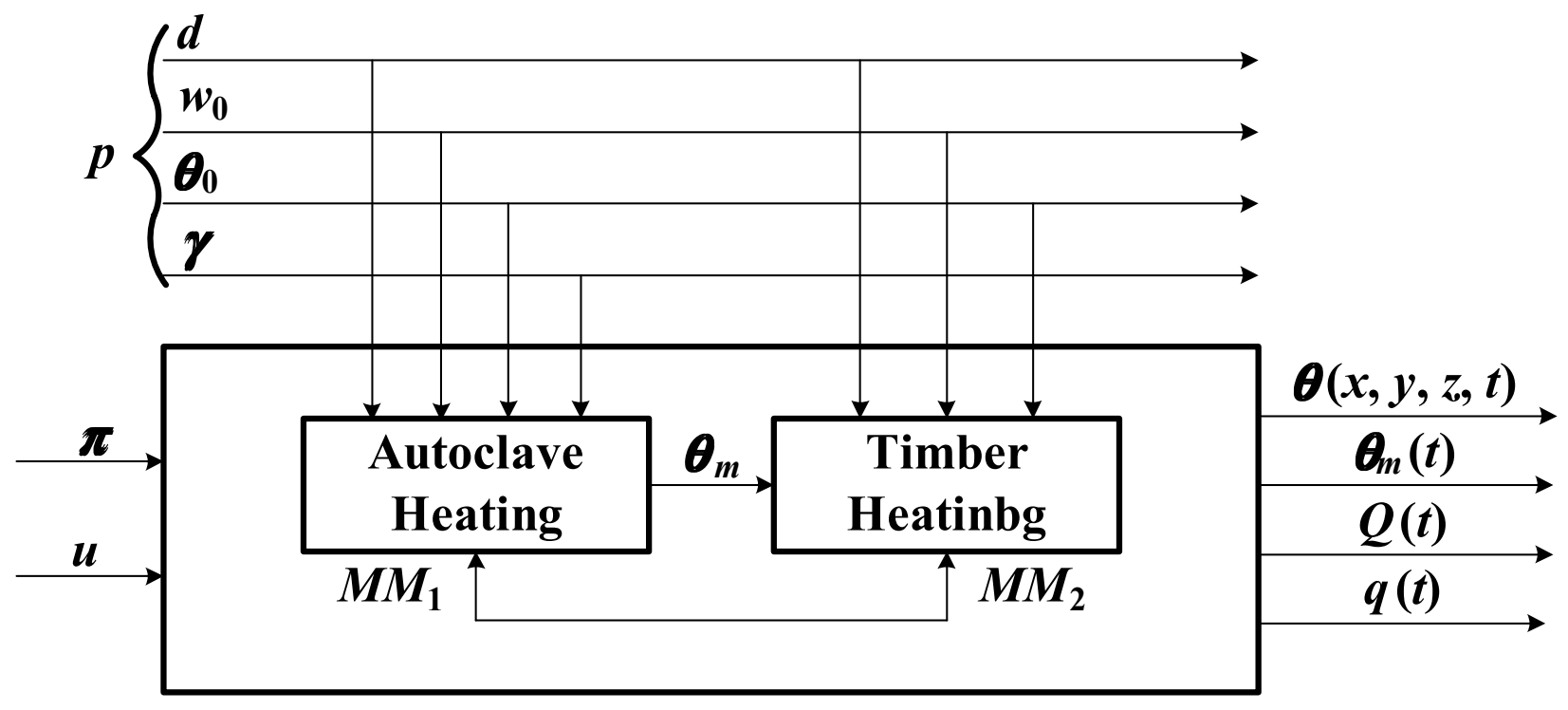

Figure 3. Scheme of unified TTP model

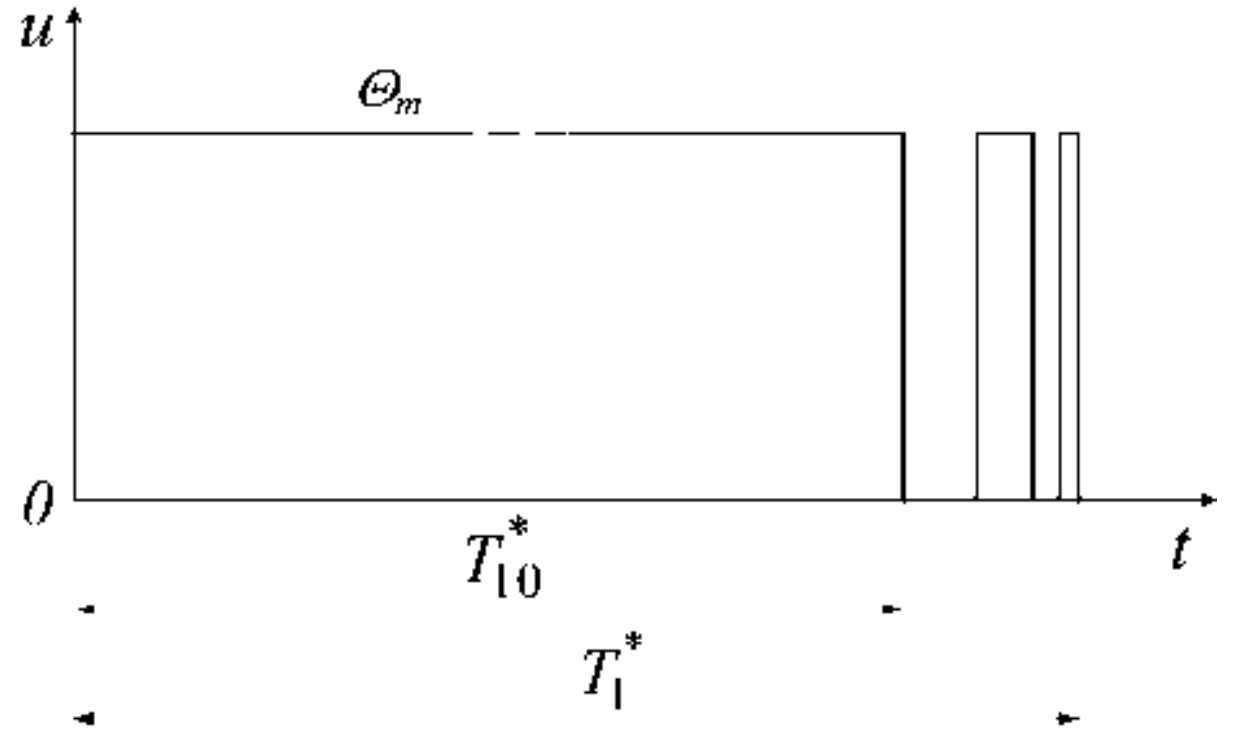

Figure 4. Minimum time control

\section{Optimization of the TTP Process by Means of Neural Networks}

In conformity with the basic investigations of the distributed-parameter-systems (DPS) time-optimal-control [13], the strict optimal control action has a form presented in figure 4.

As more than $95 \%$ of the time the temperature $\theta_{m}$ must be kept in the maximal available value $\theta_{m}{ }^{\max }$ and it is impossible to fulfill the strict re-switching $\theta_{m}{ }^{\max }-\theta_{m}^{\min }-\theta_{n n}{ }^{\max }$ due to the cooling/heating inertness, there can be used a slightly suboptimal control with active heating during the time

$$
\text { (10) } T_{1}^{*}=1.03 T_{10}{ }^{*} \text {, }
$$

where $T_{10}{ }^{*}$ is the first switching time in a strict time-optimal control [5]. For each vector parameter $p$ there is a special value of $\pi$ and the power $u$. Yet the value of the heating time $T_{1}^{*}$ has been calculated during the initial off-line simulation, 


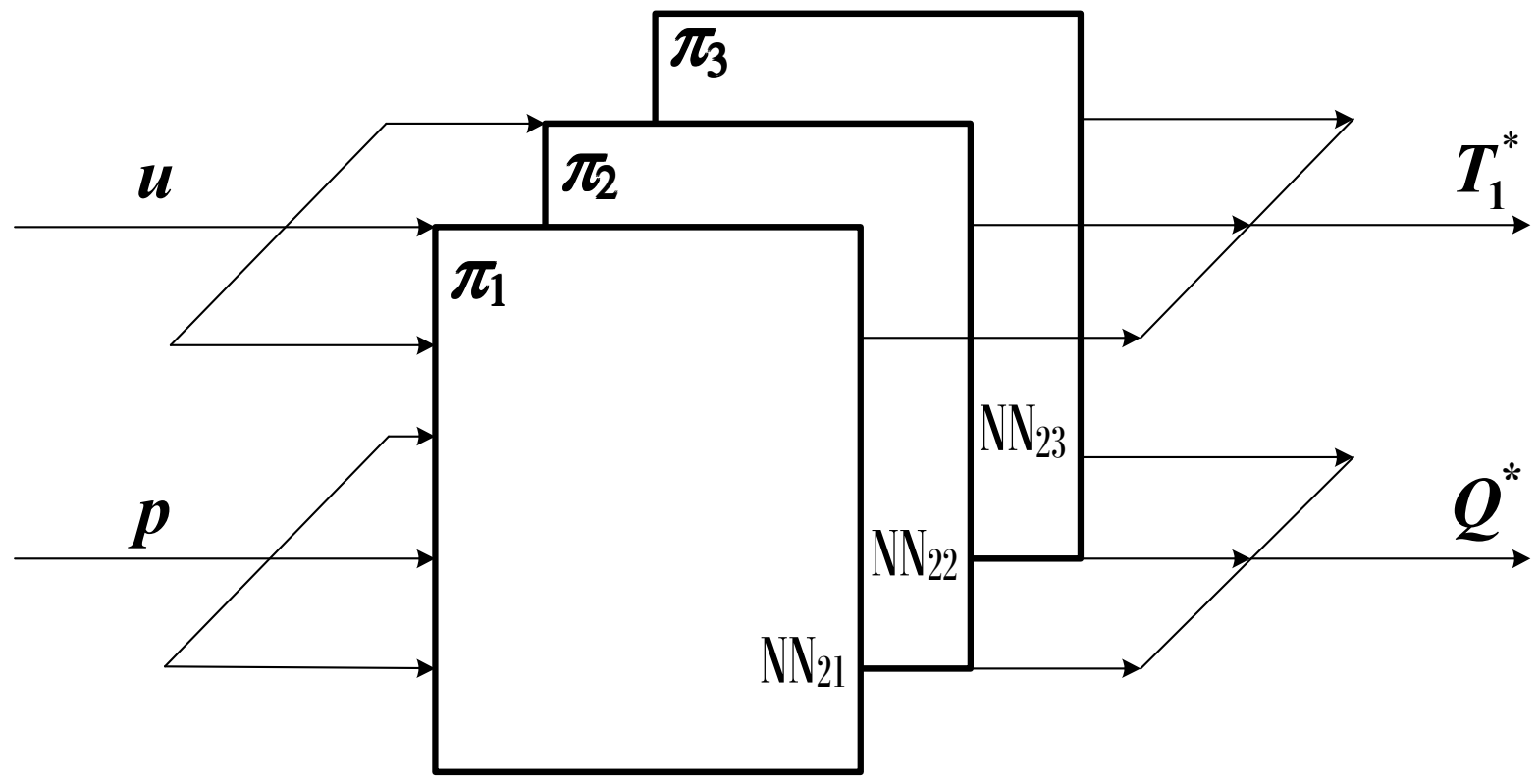

Figure 5. Static neural network $\mathrm{NN}_{\mathrm{s}}$

accomplishing the optimization procedures

$$
\text { (11) } T_{1}^{*}=T_{1}^{*}(\pi, u, p)
$$

using the set $T_{1}^{*}(\pi, u, p)$ and a static neural network $N N_{s}$ (figure 5) has been learned. Here, $Q^{*}$ is the total needed heat energy.

As the specific value of $\pi$ and the power $u^{0}$ are prescribed, the only vector $p_{j}$ represents the unknown input in the current autoclave $j$-run necessary to define the optimal heating time $T_{1}^{*}\left(p_{j}\right)$.

Following the proposed in [14] system for $p_{j}$ identification, one of the values $\bar{p}(0)$ or $\bar{p}(l)$ will be available as the input to the neural network $N N_{s}$ after finishing the estimation procedure. As the duration $T_{m}$ of the first TTP stage is many times shorter than the expected whole heating time $T$, the derived from the neural network $N N_{s}$ optimal value $T_{1}^{*}(p)$ will be accessible for the control system.

According to figure 2, the total operational time $T$ considers four components:

$$
\text { (12) } T=T_{1}+T_{2}+T_{3}+T_{4} \text {, }
$$

where $T_{1}$ is the heating time during the supply of steam from the heat generator, $T_{2}$ is the time for isochoric heating without supply of steam from the generator, $T_{3}$ and $T_{4}$ are the times of cooling timber up to an atmospheric pressure and of the air conditioning of the steamed materials respectively. All partial times $T_{i}$ depend on the different components of the parameter vector $p$. When $p$ is calculated, the values of $T_{i}(p)$ can be determined according to the results, represented in [12].

The neural network used to approximate the dependence of the optimal heating time $T_{1}{ }^{*}$ on the vector $p\left(p=\left(d, w_{0}, \theta_{0}, \gamma\right)\right)$ is a two-layer perceptron with sigmoid activation function of the neurons in the hidden layer and linear activation function of the neuron in the output layer. A set of 28 data points related to the optimal thermal treat- ment of beech materials are used for training, validation and performance test of the network. This data set is obtained by solving numerically the minimum-time problem for the TTP process based on its model (2)-(7) with different values of $p$. The results for the mean squared prediction error of the neural network with 3 hidden neurons along the epochs are shown in figure 6.

In figure 7, it is shown how the optimal heating time depends on the thickness of the wood material. Each group of 7 data points have the same value of the thickness and within each group the change of the optimal heating time is due to the influence of the other 3 parameters (humidity of the material, its temperature and the load of the autoclave).

As it can be expected, the optimal heating time increases with the increase of the thickness. The accuracy of the neural network prediction is high as it can be observed by comparing the target and the predicted data (figure 7).

\section{References}

1. Ray, W. H., D. G. Lainiotis. Distributed Parameter Pystems. New York, Marcel Dekker, 1978.

2. Christofides, P. D. Nonlinear and Robust Control of PDE Systems: Methods and Applications to Transport-Reaction Processes. Boston, Birkhäuser, 2001.

3. Dufour, P., Y. Touré, D. Blanc, P. Laurent. On Nonlinear Distributed Parameter Model Predictive Control Strategy: On-line Calculation Time Reduction and Application to an Experimental Drying Process. - Computers \& Chemical Engineering, 27, 2003, $11,1533-1542$

4. Xie, W., C. Theodoropoulos. An Off-line Model Reductionbased Technique for On-line Linear MPC Applications for Nonlinear Large-scale Distributed Systems. - Computer Aided Chemical Engineering, 28, 2010, 409-414.

5. Hadjiski, M., N. Deliiski. Advanced Control of the Wood Thermal Treatment Processing. - Cybernetics and Information 


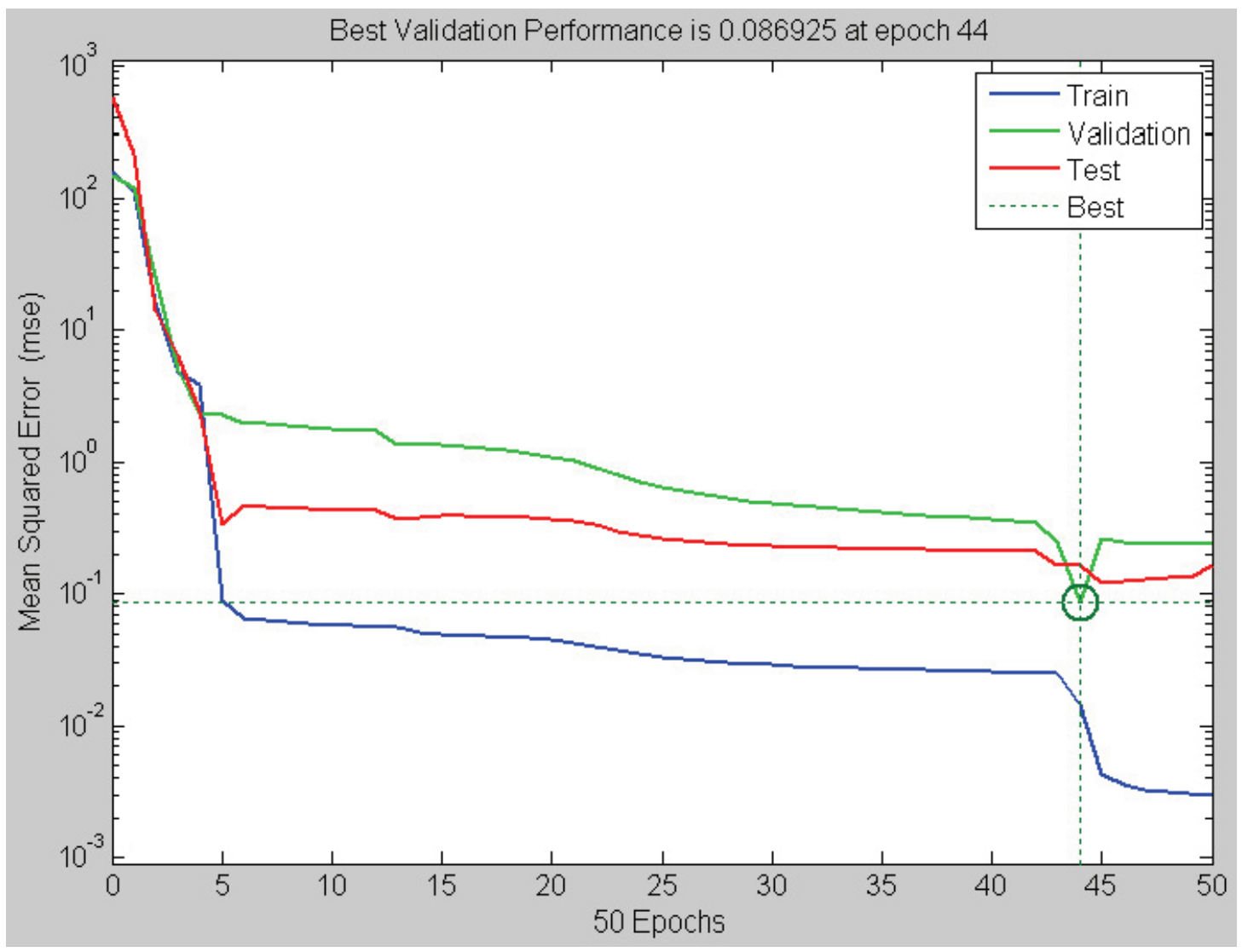

Figure 6. Mean squared prediction error along epochs

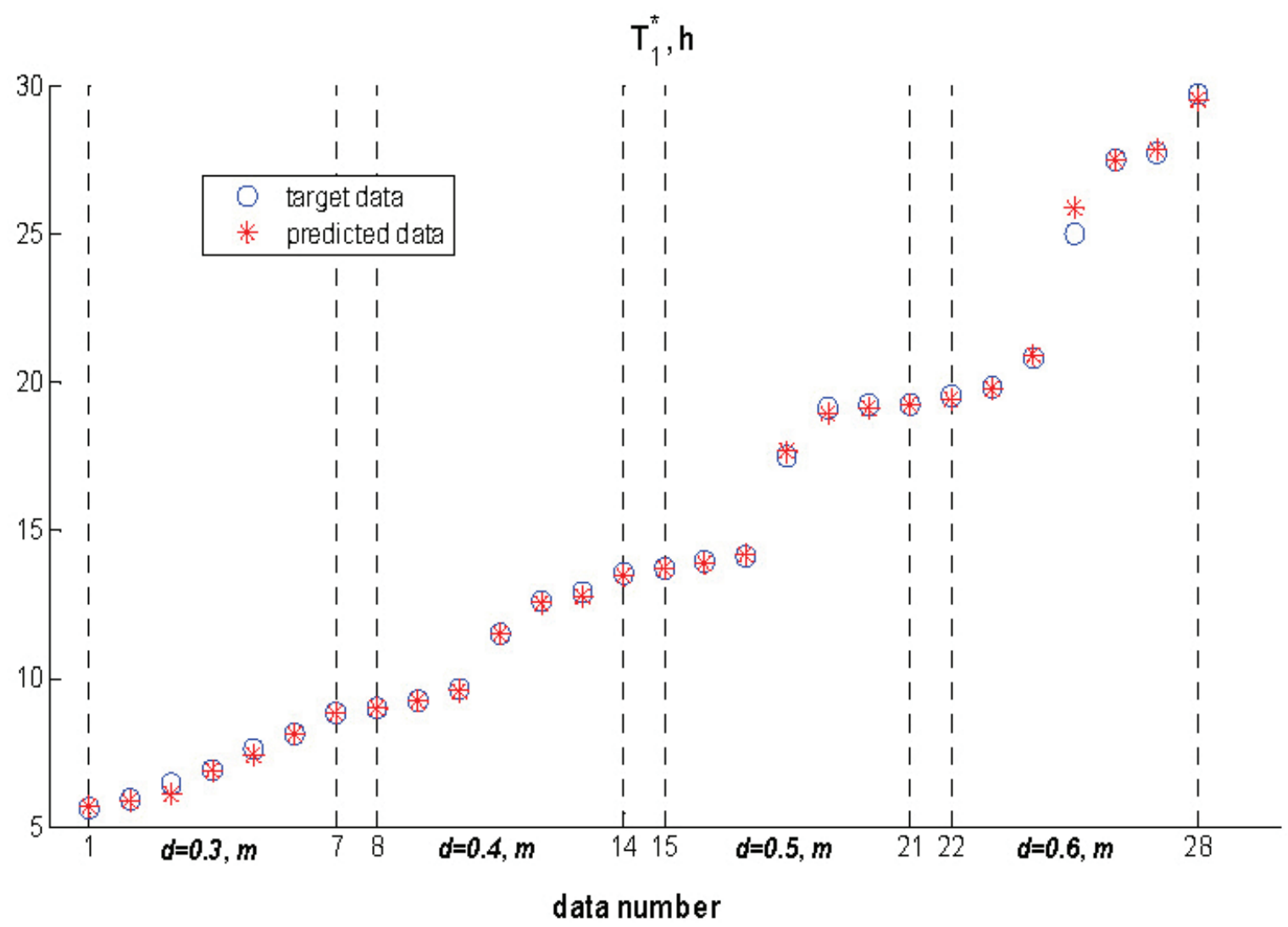

Figure 7. The dependence of the heating time on the thickness $d$ of the wood material 
Technologies, 16, 2016, No. 2, 179-197.

6. Aggelogiannaki, E., H. Sarimveis, D. Koubogiannis. Model Predictive Control in Long Ducts by Means of a Neural Network Approximation Tool. - Applied Thermal Engineering, 27, 2007, 14-15, 2363-2369.

7. Videlov, H. Drying and Thermal Treatment of Wood. University of Forestry, Sofia, 2003 (in Bulgarian).

8. Khattabi, A., H. P. Steinhagen. Update of Numerical Solution to Two-dimensional Heating of Logs. Holz als Roh- und Werkstoff, 53, 1995, 1, 93-94.

9. Younsi, R., D. Kocaefe, S. Poncsak, Y. Kocaefe. Computational Modeling of Heat and Mass Transfer During the High-temperature Heat Treatment of Wood. - Appl. Therm. Eng., 27, 2007, 1424-1431.

10. Deliiski, N. Computation of the 2-Dimensional Temperature

\section{Manuscript received on 13.06.2017}

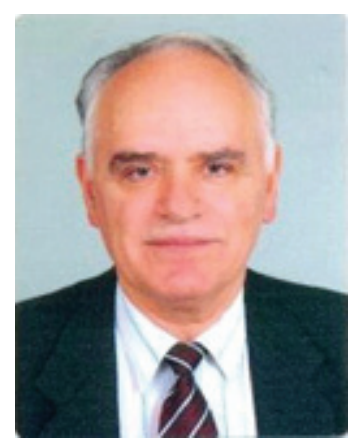

Academician Mincho Hadjiski is Professor, D.Sc. His scientific interests are in the field of mathematical modeling of technological and power processes, complex hybrid systems, incorporation of intelligence in control systems, diagnosis, inference systems, soft sensing, learning systems. He is author of 400 scientific papers and 12 books. He was supervisor of 25 Ph.D. students. He was leader of 80 international and national scientific projects and was of more than 60 IPC and NOC of international conferences. Academician Mincho Hadjiski is editor-in-chief of the journal "Automation and Informatics".

Contacts:

Institute of Information and Communication Technologies Bulgarian Academy of Science Acad. G. Bonchev St., bl. 2, 1113 Sofia e-mail:hadjiski@uctm.edu

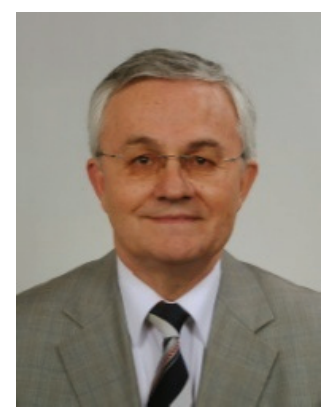

Nencho Deliiski graduated from the Forest-Technical State University in SaintPetersburg, Russia, Specialty: Automation of the Woodworking Processes, in 1970. He received a Ph.D in 1977 and a Doctor of technical sciences degree in 2003. Since 1971 he has been an Assistant, since 1984 - an Associate Professor, and since 2005 - a Professor in the Automation of Woodworking Processes, University of Forestry - Sofia. His research interests are in the area of mathematical modeling and automation of the heat- and mass transfer in the woodworking and furniture industry. He has more than 420 scientific publications in 14 countries about modeling, improvement, optimization, and automation of the technologies for thermal and hydro-thermal treatment of wood and other materials, and also in this field, he has developed more than 70 systems for automatic control, machines and installations in 22 factories around the country and abroad.

Contacts: Faculty of Forest Industry University of Forestry - Sofia 10 Kliment Ohridski Bld. 1796 Sofia

e-mail:deliiski@netbg.com
Distribution and Heat Energy Consumption of Frozen and Nonfrozen Logs. - Wood Research, 54, 2009, 3, 67-78.

11. Deliiski, N. Modelling of the Energy Needed for Heating of Capillary Porous Bodies in Frozen and Non-frozen States. Lambert Academic Publishing, Scholars' Press, Saarbrücken, Germany, 2013, 116. http://www. scholars-press.com//system/ covergenerator/build/ 1060 .

12. Deliiski, N., L. Dzurenda. Modeling of the Thermal Treatment in the Technologies for Wood Processing. University of Forestry, Sofia, 2010 (in Bulgarian).

13. Lions, J. L. Optimal Control of Systems Governed by Partial Differential Equations. Springer-Verlag, 1971.

14. Hadjiski, M., N. Deliiski, A. Grancharova. Spatiotemporal Parameter Estimation of Thermal Treatment Process via Initial Condition Reconstruction Using Neural Networks. 2017 (in Press).

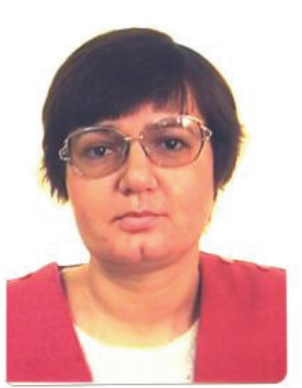

Alexandra Grancharova is Professor in Automation of Industrial Processes at the Department of Industrial Automation, University of Chemical Technology and Metallurgy (UCTM), Sofia, Bulgaria. She received her M.Sc. and Ph.D. degrees in Automation of Industrial Processes from UCTM. She co-authored the Springer monograph (Explicit Nonlinear Model Predictive Control: Theory and Applications, LNCIS, vol. 429), co-edited the Springer book (Developments in Model-Based Optimization and Control: Distributed Control and Industrial Applications, LNCIS, vol. 464) and published three textbooks for the students at UCTM (Model-based Control, Optimal and Robust Systems, Design and Analysis of Control Systems - in Bulgarian). Beside that, Grancharova has more than 120 publications in the fields of explicit model predictive control, distributed optimal control, and modeling, simulation and optimal control of industrial processes. Prof. Grancharova has been a visiting researcher and lecturer at the Norwegian University of Science and Technology in Trondheim, Jo•ef Stefan Institute in Ljubljana, University of Nova Gorica, Lund University, CentraleSupélec-Université Paris-Saclay.

Contacts:

Department of Industrial Automation University of Chemical Technology and Metallurgy

8 Kliment Ohridski Blvd.,1796 Sofia e-mail: alexandra.grancharova@abv.bg 